\title{
ARTICLE
}

\section{Colloid Formation Rates of Radionuclides Produced from Cu Foils in Water Bombarded with 120-GeV Protons}

\author{
Hiroshi MATSUMURA ${ }^{1 *}$, Shun SEKIMOTO², Hiroshi YASHIMA², Akihiro TOYODA ${ }^{1}$, Yoshimi KASUGAI ${ }^{3}$, \\ Norihiro MATSUDA ${ }^{3}$, Koji OISHI ${ }^{4}$, Kotaro BESSHO ${ }^{1}$, Yukio SAKAMOTO ${ }^{3}$, Hiroshi NAKASHIMA ${ }^{3}$, \\ David BOEHNLEIN ${ }^{5}$, Gary LAUTENSCHLAGER ${ }^{5}$, Anthony LEVELING ${ }^{5}$, Nikolai MOKHOV ${ }^{5}$, Kamran VAZIRI $^{5}$
}

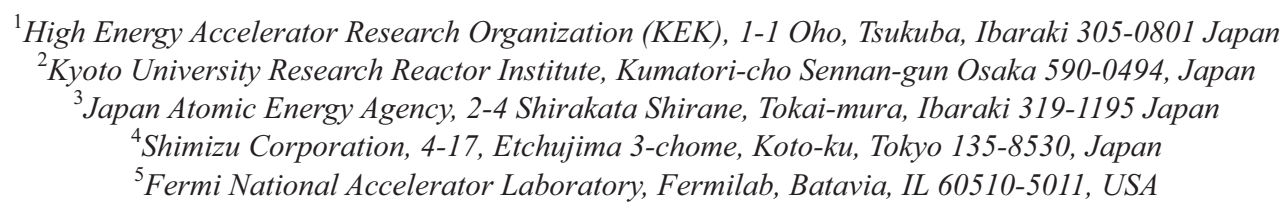

Radionuclide dissolution and radio-colloid formation in water under high-energy proton irradiation were investigated using a simple experimental apparatus. A bottle containing $\mathrm{Cu}$ foils immersed in pure water was irradiated with a $120-\mathrm{GeV}$ proton beam. After irradiation, the radioactivities of various radionuclides in the water, $\mathrm{Cu}$ foil, and colloidal and ion forms that were separated by post-irradiation ultrafiltration of the water were measured by $\gamma$-ray spectrometry. The measured radionuclides were ${ }^{7} \mathrm{Be},{ }^{24} \mathrm{Na},{ }^{28} \mathrm{Mg},{ }^{38} \mathrm{~S},{ }^{39} \mathrm{Cl},{ }^{42} \mathrm{~K},{ }^{43} \mathrm{~K},{ }^{44 \mathrm{~m}} \mathrm{Sc},{ }^{46} \mathrm{Sc},{ }^{47} \mathrm{Sc},{ }^{48} \mathrm{Sc},{ }^{48} \mathrm{Cr}$, ${ }^{52} \mathrm{Mn},{ }^{56} \mathrm{Mn},{ }^{52} \mathrm{Fe},{ }^{55} \mathrm{Co},{ }^{56} \mathrm{Co},{ }^{58} \mathrm{Co},{ }^{57} \mathrm{Ni}$, and ${ }^{61} \mathrm{Cu}$. Although the radionuclides (except for ${ }^{7} \mathrm{Be}$ produced from $\mathrm{O}$ nuclei of water) were ejected from the $\mathrm{Cu}$ foils into the water by recoil, the $\mathrm{Sc}, \mathrm{Cr}$, and $\mathrm{Fe}$ isotopes were missing from the water. The colloid formation rates of the $\mathrm{Mg}, \mathrm{Mn}, \mathrm{Co}, \mathrm{Ni}$, and $\mathrm{Cu}$ isotopes were approximately $30 \%$. The colloid formation rates of ${ }^{7} \mathrm{Be},{ }^{24} \mathrm{Na},{ }^{42} \mathrm{~K}$, and ${ }^{43} \mathrm{~K}$ were $1.8-3.9 \%$. We conclude that the radionuclide dissolution depends on the physical process and that radionuclide behavior after ejection into the water strongly depends on elemental characteristics. The cross-section ratio of ${ }^{3} \mathrm{H}$ and ${ }^{7} \mathrm{Be}$ in the water was also measured.

KEYWORDS: colloid, radionuclide, cooling water, accelerator, copper, proton, recoil, tritium

\section{Introduction}

Cooling-water systems for high energy accelerators at $\mathrm{J}_{-P A R C}{ }^{1)}$ in Japan are drained and refilled at regular intervals. Radionuclides such as ${ }^{7} \mathrm{Be}$ and ${ }^{22} \mathrm{Na}$ are produced in the cooling water or accelerator components by nuclear reactions. The radionuclides are then dissolved into the cooling water via physical and chemical processes. The radionuclides in the cooling water must be collected in demineralizers in order to reduce radioactivity below the control limit for discharge. The demineralizers adsorb ions on the ion-exchange resins. However, when radionuclides partially form radio-colloids, these radio-colloids are not efficiently captured by ion-exchange resins. This inefficient capture results in unsatisfactory recovery of the radioactivity by the demineralizers. In a previous study, we observed the weak retention of radio-colloids in a demineralizer in the case of the cooling-water system of the $\mathrm{K} 2 \mathrm{~K}$ target and magnetic horns at KEK in Japan. ${ }^{2)}$

Information on colloid formation of radionuclides in cooling water in high-energy accelerators is important to control the radioactivity. However, this phenomenon is not well understood owing to the lack of experimental data. Therefore, in this study, radionuclide dissolution and

*Corresponding Author, E-mail: hiroshi.matsumura@kek.jp (C) 2012 Atomic Energy Society of Japan, All Rights Reserved. radio-colloid formation in water were investigated through the use of a simple experimental apparatus. Because cooling water in radiation fields of high-energy accelerators is often in contact with metallic copper, which is used to fabricate accelerator components, water-immersed $\mathrm{Cu}$ foils were irradiated with $120-\mathrm{GeV}$ protons. The dissolution of radionuclides from $\mathrm{Cu}$ in water and the colloid formation rates of the radionuclides were clarified under these conditions.

Tritium is not of concern for colloid formation in water. However, in the operation of cooling-water systems, ${ }^{3} \mathrm{H}$ activity is a good indicator for radiation exposure history of water. Because both ${ }^{3} \mathrm{H}$ and ${ }^{7} \mathrm{Be}$ are mostly produced from $\mathrm{O}$ nuclei of water, cross-section ratio of ${ }^{3} \mathrm{H}$ and ${ }^{7} \mathrm{Be}$ in water is available for estimation of the residual rates of ${ }^{7} \mathrm{Be}$ in the cooling water used in the operation of high-energy accelerator facilities. Therefore, in this study, we also measured the cross-section ratio of ${ }^{3} \mathrm{H}$ and ${ }^{7} \mathrm{Be}$ in the water for the future investigation.

\section{Experiment \\ 1. Target Preparation}

Two $15 \mathrm{~mm} \times 300 \mathrm{~mm}$ sheets were cut from a $\mathrm{Cu}$ foil (purity; $99.9 \%$ and thickness; $0.03 \mathrm{~mm}$ ). The cut $\mathrm{Cu}$ foils were folded in a zigzag pattern and shaped into $15 \mathrm{~mm} \times 14$ 


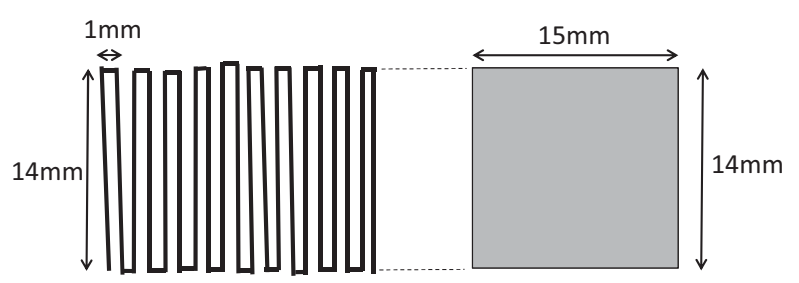

Fig. 1 Shape of folded $\mathrm{Cu}$ foil.

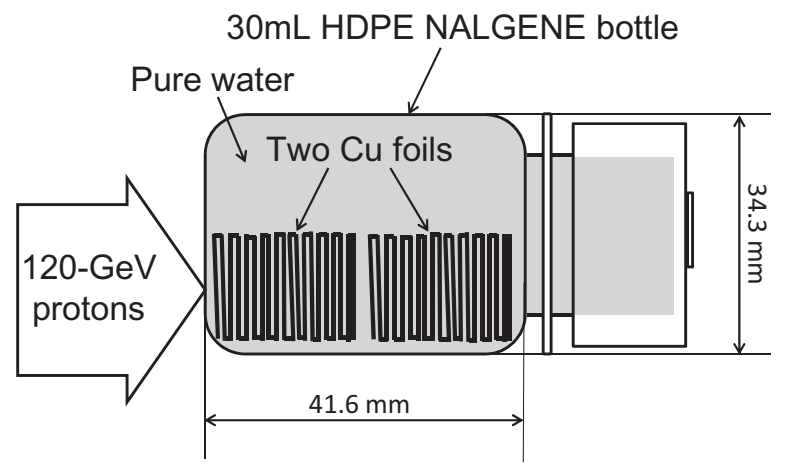

Fig. 2 Proton-irradiated water sample containing $\mathrm{Cu}$ foils.

$\mathrm{mm}$ springs, as shown in Figure 1. The $\mathrm{Cu}$ foils were designated as $\mathrm{Cu}-\mathrm{T} 1$ (weight: $1.16 \mathrm{~g}$ ) and $\mathrm{Cu}-\mathrm{T} 2$ (weight: $1.19 \mathrm{~g}$ ) in this study. The thickness of the prepared $\mathrm{Cu}$ foils was $13.6 \mathrm{mg} / \mathrm{cm}^{2}$.

The folded $\mathrm{Cu}$ foils were inserted into a $30-\mathrm{mL}$ high-density polyethylene (HDPE) bottle made by NALGENE, as shown in Figure 2. The HDPE bottle containing the $\mathrm{Cu}$ foils was filled with pure water. The weight of the water was $38.20 \mathrm{~g}$. The $\mathrm{Cu}$ foils were immersed in the pure water $12.82 \mathrm{~h}$ before proton irradiation. The bottle was sealed in a polyethylene bag and used for proton irradiation.

\section{Proton Irradiation}

The HDPE bottle containing the $\mathrm{Cu}$ foils immersed in pure water was installed into the proton beamline at M01 of the Fermi National Accelerator Laboratory (Fermilab), USA. The sample was arranged so that the center of the proton beam would penetrate the center of the $\mathrm{Cu}$ foils, as shown in Fig. 2. The sample was irradiated with a $120-\mathrm{GeV}$ proton beam for $3.250 \mathrm{~h}$. The average intensity of the proton beam was measured using a beam current monitor to be $9.69 \times 10^{8}$ protons/s. The size and position of the proton beam were previously measured by pre-irradiating a film that changed color when hit by the beam. The diameter of the beam was $\sim 2.5 \mathrm{~cm}$, which was larger than the $\mathrm{Cu}$ foils. A part of the proton beam passed through under the sample without irradiating it.

\section{Measurement of Radionuclide Activity}

The irradiated HDPE bottle was opened 50 min after of proton irradiation, and the sample was separated into several fractions within $40 \mathrm{~min}$. The scheme of sample separation after proton irradiation and the fraction names are shown in Figure 3. After the separation, the activities of (1) radionuclides in the water, (2) radionuclides that formed ions

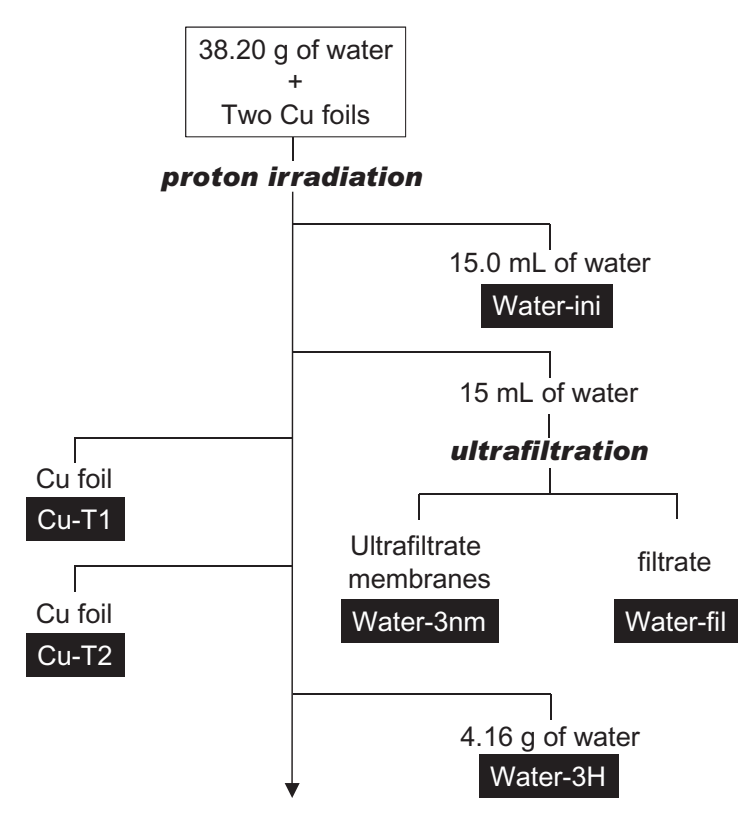

Fig. 3 Scheme of sample separation after proton irradiation. The white letters on black background denote sample names.

and colloids in the water, (3) radionuclides in the $\mathrm{Cu}$ foil, and (4) ${ }^{3} \mathrm{H}$ in the water were determined. Details on how each activity was determined are described in the following subsections.

The radioactivities of ${ }^{7} \mathrm{Be},{ }^{24} \mathrm{Na},{ }^{28} \mathrm{Mg},{ }^{38} \mathrm{~S},{ }^{39} \mathrm{Cl},{ }^{42} \mathrm{~K},{ }^{43} \mathrm{~K}$, ${ }^{44 \mathrm{~m}} \mathrm{Sc},{ }^{46} \mathrm{Sc},{ }^{47} \mathrm{Sc},{ }^{48} \mathrm{Sc},{ }^{48} \mathrm{Cr},{ }^{52} \mathrm{Mn},{ }^{56} \mathrm{Mn},{ }^{52} \mathrm{Fe},{ }^{55} \mathrm{Co},{ }^{56} \mathrm{Co}$, ${ }^{58} \mathrm{Co},{ }^{57} \mathrm{Ni}$, and ${ }^{61} \mathrm{Cu}$ in the fractions were determined by $\gamma$-ray spectrometry with an HPGe detector previously calibrated by Canberra ${ }^{3)}$. The detector efficiencies were determined by using Canberra's LabSOCS software ${ }^{4)}$. Nuclear data in the literature ${ }^{5)}$ were used for data analysis.

\section{(1) Radionuclides in Water}

From the proton-irradiated HDPE bottle, $15.0 \mathrm{~mL}$ of water was transferred into a $15-\mathrm{mL}$ low-density polyethylene (LDPE) bottle by using a $5000-\mu \mathrm{L}$ piston-driven air displacement pipette. The LDPE bottle was designated as Water-ini. In order to acidify the water, $0.100 \mathrm{~mL}$ of $6 \mathrm{M}$ $\mathrm{HNO}_{3}$ was added to Water-ini. The radioactivities at the end of proton irradiation $\left(A_{0}\right)$ in Water-ini were then determined by $\gamma$-ray spectrometry.

(2) Radionuclides Forming Colloids and Ions in Water

An ultrafiltration unit (Sartorius Stedim, Vivaspin $20 \mathrm{ml}$, 10,000 MWCO) for concentration and/or purification of biological samples was used to separate colloids and ions in water. The ultrafiltration unit consisted of a concentrator (upper part) and filtrate vessel (lower part). The concentrator was equipped with two polyethersulfone ultrafiltration membranes (pore size: $\sim 3 \mathrm{~nm}$ ). In order to let all the water pass through the ultrafiltration membranes, a concentration pocket on the bottom of the concentrator was filled up with a Teflon bar. From the proton-irradiated HDPE bottle, $15.0 \mathrm{~mL}$ of water was then transferred into the concentrator of the ultrafiltration unit by using the $5000-\mu \mathrm{L}$ piston-driven air displacement pipette. The ultrafiltration unit was centrifuged 


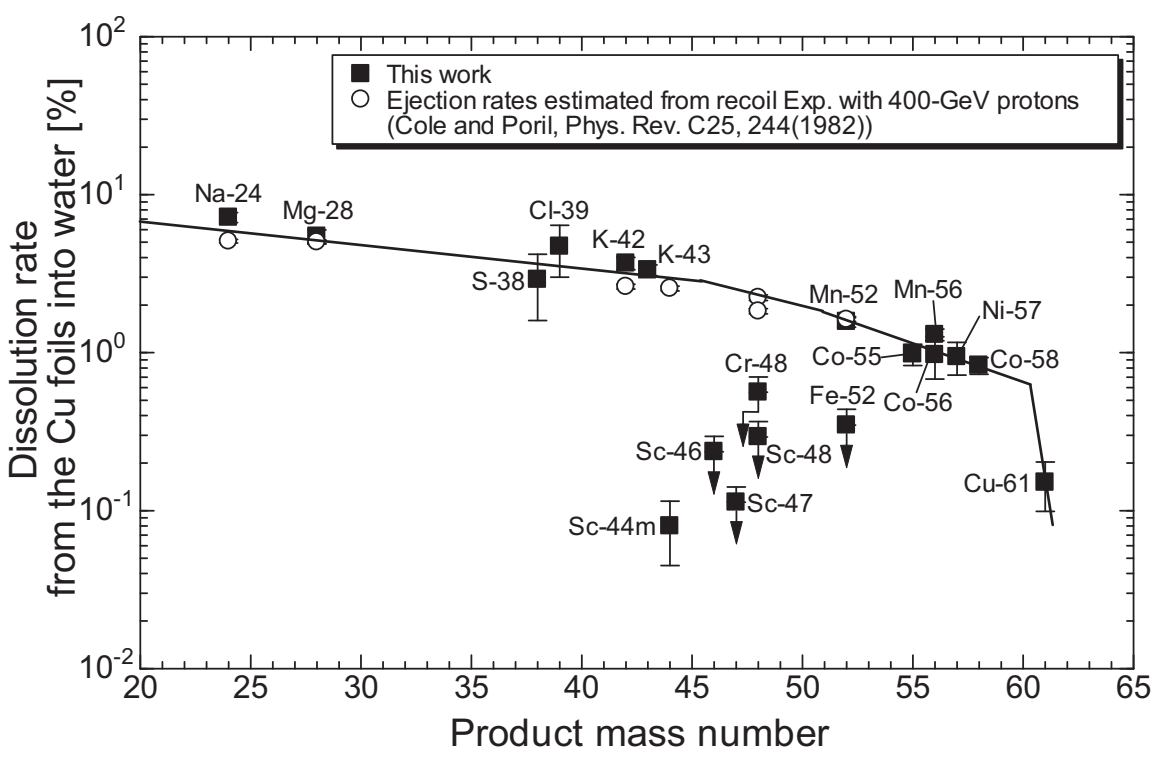

Fig. 4 Dissolution rates of radionuclides from $\mathrm{Cu}$ foils.

The closed squares with an arrow indicate upper limits of the rates.

at $4000 \mathrm{rpm}$ for $10 \mathrm{~min}$. Colloids larger than $\sim 3 \mathrm{~nm}$ were trapped by the ultrafiltration membranes, and the filtrate was collected in the filtrate vessel.

After centrifuging, the two ultrafiltration membranes were peeled off the unit with a cutter and transferred into a polyethylene bag. This sample was designated as Water-3nm. The $A_{0}$ in Water-3nm was determined by $\gamma$-ray spectrometry.

The filtrate collected in the filtrate vessel was transferred into a 15-mL LDPE bottle designated as Water-fil. In order to acidify the filtrate, $0.100 \mathrm{~mL}$ of $6 \mathrm{M} \mathrm{HNO}_{3}$ was added to Water-fil. The $A_{0}$ in Water-fil was then determined by $\gamma$-ray spectrometry.

\section{(3) Radionuclides in $\mathrm{Cu}$ Foils}

The $\mathrm{Cu}$ foils were picked up from the HDPE bottle with tweezers. The $\mathrm{Cu}$ foil designated as $\mathrm{Cu}-\mathrm{T} 1$ was wiped, dried, crushed flat, and sealed into a polyethylene bag. The $A_{0}$ in $\mathrm{Cu}$-T1 was then determined by $\gamma$-ray spectrometry.

(4) Tritium in Water

The last fraction of Water-3H was used to measure ${ }^{3} \mathrm{H}$ activity. For this, $2.5 \mathrm{~mL}$ of Water-3H was mixed with $10 \mathrm{~mL}$ of a scintillation cocktail. Radioactivity of the tritium was then determined by a liquid scintillation counter.

\section{Results and Discussion}

\section{Dissolution Rates of Radionuclides from $\mathrm{Cu}$}

The total radioactivities in the water and $\mathrm{Cu}$ foils were estimated from $A_{0}$ measured in Water-ini and $\mathrm{Cu}-\mathrm{T} 1$, respectively. The dissolution rates of the radionuclides from the $\mathrm{Cu}$ foils into the water were calculated by using these estimated total radioactivities.

The dissolution rates of the radionuclides (except for ${ }^{7} \mathrm{Be}$ produced from $\mathrm{O}$ nuclei of water) from the $\mathrm{Cu}$ foils were plotted with the estimated recoil ejection rates, as shown in Figure 4. The closed squares indicate the dissolution rates measured in this study. The solid line indicates the trend of the measured dissolution rates obtained by the eye-guide. The open circles indicate ejection rates of the radionuclides from the $\mathrm{Cu}$ foils into the water as estimated from the recoil property of using the experimental mean ranges in a $\mathrm{Cu}$ target material $(2 W(F+B))$, which was from the literature ${ }^{6}$.

The measured dissolution rates of most of the radionuclides agreed with the ejection rates estimated by recoil. This indicates that the radionuclides were predominantly ejected from the $\mathrm{Cu}$ foils into the water by the physical phenomenon. On the other hand, the dissolution rates of ${ }^{44 \mathrm{~m}} \mathrm{Sc},{ }^{46} \mathrm{Sc},{ }^{47} \mathrm{Sc},{ }^{48} \mathrm{Sc},{ }^{48} \mathrm{Cr}$, and ${ }^{52} \mathrm{Fe}$ were much lower than the ejection rates estimated by recoil. These radionuclides must also be in the water according to the recoil property because the physical process cannot be avoided. However, the radionuclides were missing from the water. Under these conditions, the main body of the colloids may be copper hydroxides dissolved from the $\mathrm{Cu}$ foils. Therefore, the missing elements may be immediately adsorbed onto the $\mathrm{Cu}$ foils and/or HDPE bottle surface without adsorption onto the copper hydroxide colloids after ejection into the water. This behavior depends on elemental characteristics because all scandium isotopes were missing from the water together.

\section{Colloid Formation Rates of Radionuclides in Water}

Colloid rates of the radionuclides in water after proton irradiation were obtained by using $A_{0}$ in Water-3nm and Water-ini. Ion rates of the radionuclides in water after proton irradiation were obtained by using $A_{0}$ in Water-fil and Water-ini. Both the colloid rates and ion rates of ${ }^{46} \mathrm{Sc}$, ${ }^{48} \mathrm{Sc},{ }^{48} \mathrm{Cr}$, and ${ }^{52} \mathrm{Fe}$ could not be obtained since they were missing from the water. Figure 5 shows the colloid rates (gray bars) and ion rates (white bars) of the radionuclides in water. The sum of the measured colloid rate and ion rate for each radionuclide was approximately $100 \%$ within error. Therefore, the colloid and ion rates for each radionuclide 


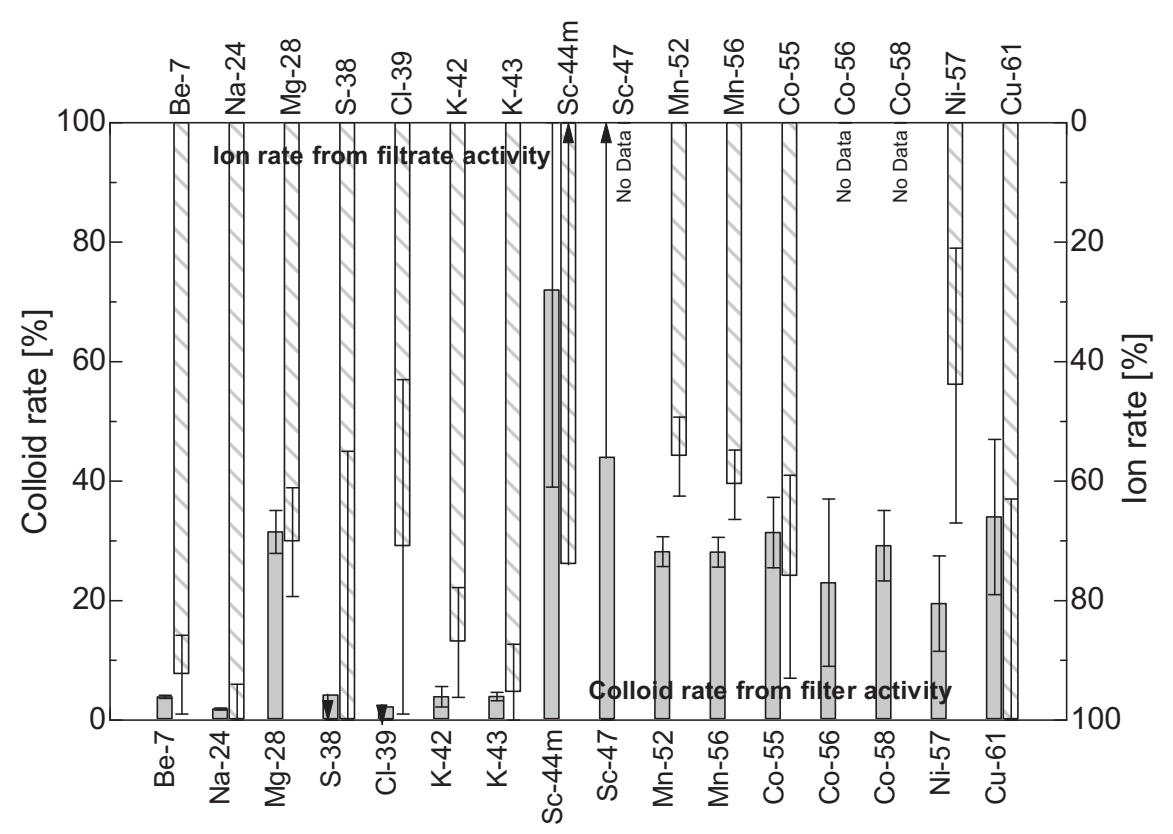

Fig. 5 Colloid rates and ion rates of radionuclides in water. The arrows indicate upper or lower limits of the rates.

were consistent with each other.

The colloid rates of ${ }^{28} \mathrm{Mg},{ }^{52} \mathrm{Mn},{ }^{56} \mathrm{Mn},{ }^{55} \mathrm{Co},{ }^{56} \mathrm{Co},{ }^{58} \mathrm{Co}$, ${ }^{57} \mathrm{Ni}$, and ${ }^{61} \mathrm{Cu}-$ which are susceptible to hydroxides-were similar and showed relatively high rates of about $30 \%$. The colloid rates of ${ }^{44 \mathrm{~m}} \mathrm{Sc}$ and ${ }^{47} \mathrm{Sc}$, which were one of the missing elements in the water, were quite higher than those for the others. The colloid rates of ${ }^{7} \mathrm{Be},{ }^{24} \mathrm{Na},{ }^{42} \mathrm{~K}$, and ${ }^{43} \mathrm{~K}$ were similar at $1.8-3.9 \%$. Although ${ }^{7} \mathrm{Be}$ is also susceptible to hydroxides, the colloid rate was relatively low. However, the radioactivity of ${ }^{7} \mathrm{Be}$ directly produced in cooling water is significantly higher than those of the others. Therefore, the colloid formation of ${ }^{7} \mathrm{Be}$ causes serious problems for radioactivity collection despite the low colloid formation rates.

\section{Cross-section Ratio of ${ }^{3} \mathrm{H}$ and ${ }^{7} \mathrm{Be}$ in Water}

The cross-section ratio for ${ }^{3} \mathrm{H}$ and ${ }^{7} \mathrm{Be}$ productions $\left({ }^{3} \mathrm{H} /{ }^{7} \mathrm{Be}\right)$ in the water was measured as $6.1 \pm 0.3$. This ratio is available for estimation of the residual rates of ${ }^{7} \mathrm{Be}$ in cooling water used in the operation of high-energy accelerator facilities in the future investigation.

\section{Conclusions}

In water with $\mathrm{Cu}$ foils irradiated by $120-\mathrm{GeV}$ protons, most radionuclides were ejected from the $\mathrm{Cu}$ foils into the water by recoil. The $\mathrm{Sc}, \mathrm{Cr}$, and $\mathrm{Fe}$ isotopes were missing from the water even though they were ejected into the water. The colloid formation rates of the $\mathrm{Mg}, \mathrm{Mn}, \mathrm{Co}, \mathrm{Ni}$, and $\mathrm{Cu}$ isotopes were found to be about $30 \%$. The colloid formation rates of ${ }^{7} \mathrm{Be},{ }^{24} \mathrm{Na},{ }^{42} \mathrm{~K}$, and ${ }^{43} \mathrm{~K}$ were found to be $1.8-3.9 \%$. We conclude that dissolution of radionuclides depends on a physical process and that radionuclide behavior after ejection into water strongly depends on chemical characteristics.
Furthermore, the cross-section ratio of ${ }^{3} \mathrm{H}$ and ${ }^{7} \mathrm{Be}$ productions in water was found to be $6.1 \pm 0.3$ in $120-\mathrm{GeV}$ proton irradiation.

\section{Acknowledgment}

This work was supported by a grant-in-aid from the Ministry of Education, Science and Culture (KAKENHI 19360432) in Japan. Fermilab is a US Department of Energy Laboratory operated under Contract E-AC02-07CH11359 by the Fermi Research Alliance, LLC.

\section{References}

1) Accelerator Group JAERI/KEK Joint Project Team, "Accelerator technical design report for high-intensity proton accelerator facility project, J-PARC," KEK Report 2002-13, JAERI Tech 2003-044, J-PARC 03-01 (2003).

2) H. Matsumura, N. Kinoshita, A. Toyoda, K. Masumoto, K. Bessho, M. Hagiwara, Y. Yamanoi, "Adsorption behavior of radionuclides on ion-exchange resin from cooling water for the K2K target and magnetic horns," Nuclear Technology, 168, 979-983 (2009).

3) R. Venkataraman, F. Bronson, V. Atrashkevich, M. Field, B. M. Young, "Improved detector response characterization method in ISOCS and LabSOCS," $J$. Radioanal. Nucl. Chem., 264, 213 (2005)

4) F. L. Bronson, "Validation of the accuracy of the LabSOCS software for mathematical efficiency calibration of Ge detectors for typical laboratory samples," J. Radioanal. Nucl. Chem., 255, 137 (2003).

5) R. B. Firestone, Table of Isotopes, 8th ed., John Wiley and Sons, Inc., New York (1996).

6) G. D. Cole and N. T. Porile, "Recoil properties of fragments emitted in the interaction of complex nuclei with relativistic ${ }^{12} \mathrm{C}$ ions and protons," Phys. Rev. C25, 244 (1982). 